\title{
PECTIN; HETERO POLYSACCHARIDE AS A GREEN AND NATURAL CATALYST FOR THE SYNTHESIS OF DIHYDRO-2-OXOPYRROLES AND 3,4,5-TRISUBSTITUTED FURAN-2(5H)-ONES
}

\author{
MEHRNOOSH KANGANI, NOURALLAH HAZERI*, MALEK-TAHER MAGHSOODLOU \\ Department of Chemistry, Faculty of Sciences, University of Sistan and Baluchestan, P.O. Box 98135-674,Zahedan, Iran
}

\begin{abstract}
Use of green and biodegradable catalyst in organic synthesis is undeniable. Easy access, inexpensive, natural and green catalysts are valuable. In this study, a green procedure by using pectin as a green and natural catalyst for the one-pot synthesis of dihydro-2-oxopyrroles and 3,4,5-trisubstituted furan-2(5H)-ones at ambient temperature in aqueous media has been developed. This methodology has some advantages such as: use of a hetero polysaccharide as a easy access and biodegradable catalyst, clean work-up and no need to column chromatography.
\end{abstract}

Keywords: Pectin; dihydro-2-oxopyrroles; 3,4,5-trisubstituted furan-2(5H)-ones

\section{INTRODUCTION}

Pectin is one of the major plant cell wall components and probably the most complex macromolecule in nature, as it can be composed out of as many as 17 different monosaccharides containing more than 20 different linkages [1-3]. Pectin is used in foods mainly as gelling, stabilizing, or thickening agent in products such as jam, yoghurt drinks, fruity milk drinks, and ice cream [4] Most of the pectin used by food industry originates from citrus or apple peel from which it is extracted at low $\mathrm{pH}$ and high temperature and is primarily a homogalacturonan [5]. In products that naturally contain pectin, e.g., fruit and vegetables, important quality changes during storage and processing are related to changes in pectin structure. Native or added pectic enzymes can play an important role in these changes [6] (Figure 1).

Dihydropyrroles are important azaheterocycles with various biological activities [7]. For example, dihydropyrrole derivatives have been used as the inhibitors of bacterial peptide deformylase [8], human mitotic kinesin Eg5 [9] cardiac CAMP phosphodiesterase [10], human immunodeficiency virus (HIV) integrase [11], and vascular endothelial growth factor receptors (VEGFR) [12] . They are also useful intermediates in the synthesis of natural products 6 and chemicals [13] . Thus, the development of efficient synthetic methodologies for novel dihydropyrroles has attracted tremendous interests in synthetic chemistry. Moreover, the dihydropyrrol-2-one unit is the core structure in a number of natural products [14-17]. As a result, considerable efforts have been made for the synthesis of this important class of compounds [18-24]
Recently, the most convenient methods have been reported for preparation of the dihdropyrrol-2-ones by means of domino reaction between amines, dialkyl acetylenedicarboxylates and aldehydes $[25,26]$. With widespread applications and bioactivity, development of efficient approach to dihdropyrrol-2-ones in terms of mild reaction conditions, operational simplicity, and readily available material is still in demand.

Sweeney et al. reported the first preparation of 3,4-bistributylstannyl $2(5 \mathrm{H})$-furanones by reacting TBS as well as THP protected butynoate with hexabutylditin in the presence of $\mathrm{PdCl}_{2}\left(\mathrm{PPh}_{3}\right)_{2}$ leading to substituted acrylate intermediate, which upon treating under a variety of conditions yielded desired furanone synthon [27-29]. More recently Mauro et al. described the construction of furanone system via ring-closing metathesis catalyzed by the first generation Grubbs' catalyst [30] . However, some of these catalysts suffer from the drawback of green chemistry such as prolonged reaction times, low yields, toxicity and recovery and reusability of the catalyst. Therefore, introducing clean processes and utilizing eco-friendly and green catalysts which can be simply worked-up at the end of reactions have been under permanent attention.

Therefore, In continuation to our greener method development program [31-37], we have found hetero polysaccharide pectin as a green catalyst which is cheap and easily available. We investigated its potential for catalyzing the synthesis of dihydro-2-oxopyrroles and 3,4,5-trisubstituted furan-2(5H)ones (Scheme 1) and the results are presented in this paper. The process is remarkably simple, high yielding, highly efficient, time and energy saving.

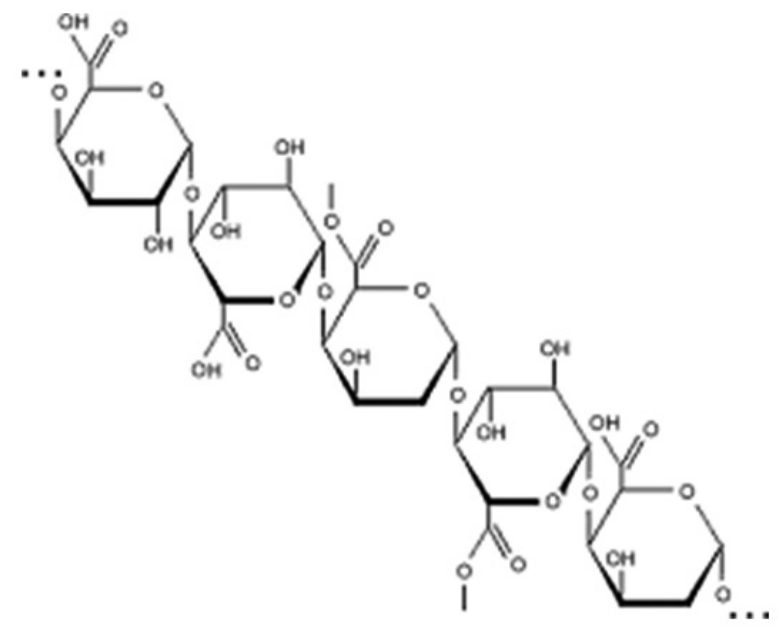

Figure 1.The structure of pectin. 


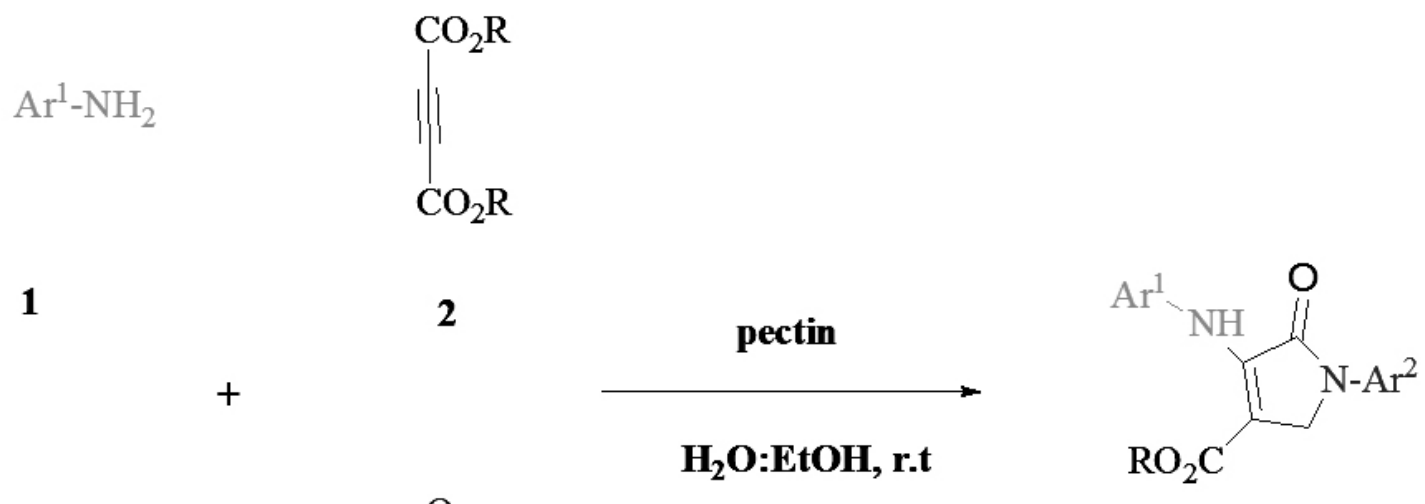

$\mathrm{Ar}^{2}-\mathrm{NH}_{2}$<smiles>C=O</smiles><smiles>[R20]C(=O)C#CC([R2])[R]</smiles>

7

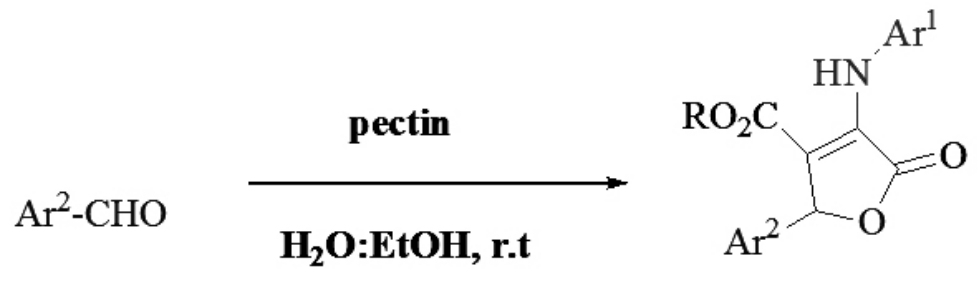

8
9

Scheme 1. Synthesis of dihydro-2-oxopyrroles and 3,4,5-trisubstituted furan-2(5H)-ones in the presence of pectin as green and natural catalyst at ambient temperature in aqueous media.

\section{RESULTS AND DISCUSSION}

The reaction of aniline, dimethylacetylendicarboxylate and formaldehyde was chosen as a model system for optimization the reaction conditions. The reaction was performed with different amount of solvent and catalyst. The results are summarized in table 1 and table2. Since we wanted to present a green and environmentally benign protocol for this experiment, we did not test any other organic solvents under these conditions.

Table 1. Effect of amount of catalyst for the synthesis of dihydro-2oxopyrroles.

\begin{tabular}{|c|c|c|c|}
\hline Entry & Catalyst loading $(\mathrm{g})$ & Time $(\mathrm{min})$ & Yield (\%) \\
\hline 1 & 0.02 & 120 & 42 \\
\hline 2 & 0.03 & 100 & 52 \\
\hline 3 & 0.04 & 90 & 63 \\
\hline 4 & $\mathbf{0 . 0 5}$ & $\mathbf{6 0}$ & $\mathbf{7 9}$ \\
\hline 5 & 0.06 & 60 & 79 \\
\hline
\end{tabular}

Table2. Influence of different solvents for the synthesis of dihydro-2oxopyrroles in the presence of pectin $(0.05 \mathrm{~g})$ at ambient temperature.

\begin{tabular}{|c|c|c|}
\hline Entry & Solvent & Yield(\%) \\
\hline 1 & $\mathrm{H}_{2} \mathrm{O}$ & 59 \\
\hline 2 & $\mathrm{H}_{2} \mathrm{O}: \mathrm{EtOH}(1: 1)$ & 65 \\
\hline 3 & $\mathrm{H}_{2} \mathrm{O}: \mathrm{EtOH}(1: 2)$ & 68 \\
\hline 4 & $\mathbf{H}_{2} \mathbf{O}: \mathbf{E t O H}(\mathbf{1 : 3})$ & $\mathbf{7 9}$ \\
\hline 5 & $\mathrm{H}_{2} \mathrm{O}: \mathrm{EtOH}(1: 4)$ & 75 \\
\hline 6 & $\mathrm{EtOH}$ & 75 \\
\hline
\end{tabular}

Next the reaction conditions were optimized for the synthesis of 3,4,5-trisubstituted furan-2 $(5 \mathrm{H})$-ones, the best results was found at ambient temperature with $(0.05 \mathrm{~g})$ of pectin in $\mathrm{H}_{2} \mathrm{O}: \mathrm{EtOH}(1: 3)$.

The scope and efficiency of these procedures were explored for the synthesis of a wide variety of substituted dihydro-2-oxopyrroles and 3,4,5-trisubstituted furan-2(5H)-ones. A series of aromatic aldehydes and amines were investigated (Table 3). Interestingly, a variety of aryl aldehydes and amines including electron withdrawing or releasing substituents (ortho-, meta-, and para-substituted) participated well in this reaction and gave the products in good to excellent yield. 
J. Chil. Chem. Soc., 63, No 4 (2018)

Table 3. Synthesis of dihydro-2-oxopyrroles and 3,4,5-trisubstituted furan-2(5H)-ones.

\begin{tabular}{|c|c|c|c|c|c|c|c|}
\hline Product & $\mathrm{Ar}^{1}$ & $\mathrm{Ar}^{2}$ & $\mathrm{R}$ & Time (min) & yield (\%) & m.p $\left({ }^{\circ} \mathrm{C}\right)$ & Lit.mp $\left({ }^{\circ} \mathrm{C}\right)[\mathrm{Ref}]$ \\
\hline $5 \mathrm{a}$ & $\mathrm{Ph}$ & $\mathrm{Ph}$ & $\mathrm{Me}$ & 60 & 79 & $153-154$ & $155-156[26]$ \\
\hline $5 b$ & $\mathrm{Ph}$ & $\mathrm{Ph}$ & Et & 60 & 75 & $135-137$ & $138-140[25]$ \\
\hline $5 c$ & $4-\mathrm{Me}-\mathrm{C}_{6} \mathrm{H}_{4}$ & $4-\mathrm{Me}-\mathrm{C}_{6} \mathrm{H}_{4}$ & $\mathrm{Me}$ & 50 & 85 & $170-172$ & $177-178[26]$ \\
\hline $5 \mathrm{~d}$ & $4-\mathrm{Me}-\mathrm{C}_{6} \mathrm{H}_{4}$ & $4-\mathrm{Me}-\mathrm{C}_{6} \mathrm{H}_{4}$ & Et & 60 & 75 & $125-128$ & $131-132[25]$ \\
\hline $5 \mathrm{e}$ & $4-\mathrm{OMe}-\mathrm{C}_{6} \mathrm{H}_{4}$ & $4-\mathrm{OMe}-\mathrm{C}_{6} \mathrm{H}_{4}$ & Et & 120 & 80 & $151-153$ & $152-154[32]$ \\
\hline $5 f$ & $4-\mathrm{F}-\mathrm{C}_{6} \mathrm{H}_{4}$ & $4-\mathrm{F}-\mathrm{C}_{6} \mathrm{H}_{4}$ & $\mathrm{Me}$ & 60 & 85 & $160-163$ & $163-165[32]$ \\
\hline $5 \mathrm{~g}$ & $4-\mathrm{Cl}-\mathrm{C}_{6} \mathrm{H}_{4}$ & $4-\mathrm{Cl}-\mathrm{C}_{6} \mathrm{H}_{4}$ & Et & 60 & 85 & $161-163$ & $168-170[32]$ \\
\hline $5 \mathrm{~h}$ & 4- $\mathrm{Br}-\mathrm{C}_{6} \mathrm{H}_{4}$ & $4-\mathrm{Br}-\mathrm{C}_{6} \mathrm{H}_{4}$ & Et & 90 & 80 & $167-169$ & $169-171[32]$ \\
\hline $5 \mathrm{i}$ & $\mathrm{PhCH}_{2}$ & $\mathrm{Ph}$ & $\mathrm{Me}$ & 60 & 85 & $136-138$ & $140-141[25]$ \\
\hline $5 \mathrm{j}$ & $\mathrm{PhCH}_{2}$ & $\mathrm{Ph}$ & Et & 60 & 80 & 81127 & $129130[25]$ \\
\hline $5 \mathrm{k}$ & $\mathrm{PhCH}_{2}$ & $4-\mathrm{F}-\mathrm{C}_{6} \mathrm{H}_{4}$ & $\mathrm{Me}$ & 60 & 70 & $166-168$ & $166-168[32]$ \\
\hline 51 & $\mathrm{PhCH}_{2}$ & $4-\mathrm{Br}-\mathrm{C}_{6} \mathrm{H}_{4}$ & $\mathrm{Me}$ & 60 & 70 & $119-121$ & $120-121[26]$ \\
\hline $5 \mathrm{~m}$ & $\mathrm{PhCH}_{2}$ & $4-\mathrm{Me}-\mathrm{C}_{6} \mathrm{H}_{4}$ & $\mathrm{Me}$ & 60 & 75 & $144-146$ & $144-146[32]$ \\
\hline $9 \mathrm{a}$ & $\mathrm{Ph}$ & $\mathrm{Ph}$ & $\mathrm{Me}$ & 120 & 75 & $157-1158$ & $159-162[30]$ \\
\hline $9 b$ & $\mathrm{Ph}$ & $4-\mathrm{Me}-\mathrm{C}_{6} \mathrm{H}_{4}$ & $\mathrm{Me}$ & 70 & 80 & $281-283$ & $284-287[30]$ \\
\hline $9 \mathrm{c}$ & $4-\mathrm{Me}-\mathrm{C}_{6} \mathrm{H}_{4}$ & $\mathrm{Ph}$ & $\mathrm{Me}$ & 60 & 85 & $185-186$ & $181-183$ [30] \\
\hline $9 \mathrm{~d}$ & $4-\mathrm{Cl}-\mathrm{C}_{6} \mathrm{H}_{4}$ & $\mathrm{Ph}$ & $\mathrm{Me}$ & 60 & 85 & $150-152$ & $149-152[30]$ \\
\hline $9 \mathrm{e}$ & $4-\mathrm{CN}-\mathrm{C}_{6} \mathrm{H}_{4}$ & $\mathrm{Ph}$ & Et & 40 & 80 & $150-152$ & $152-154$ [37] \\
\hline $9 f$ & $\mathrm{Ph}$ & $4-\mathrm{F}-\mathrm{C}_{6} \mathrm{H}_{4}$ & $\mathrm{Me}$ & 50 & 85 & $290-293$ & $293-295$ [32] \\
\hline $9 \mathrm{~g}$ & $\mathrm{Ph}$ & $4-\mathrm{Cl}-\mathrm{C}_{6} \mathrm{H}_{4}$ & $\mathrm{Me}$ & 60 & 85 & $167-170$ & $165-166[32]$ \\
\hline $9 \mathrm{~h}$ & $\mathrm{Ph}$ & $3-\mathrm{NO}_{2}-\mathrm{C}_{6} \mathrm{H}_{4}$ & $\mathrm{Me}$ & 90 & 80 & $199-202$ & $203-205$ [32] \\
\hline $9 \mathrm{i}$ & $4-\mathrm{OMe}-\mathrm{C}_{6} \mathrm{H}_{4}$ & $\mathrm{Ph}$ & $\mathrm{Me}$ & 60 & 85 & $235-238$ & $239-242$ [32] \\
\hline $9 \mathrm{j}$ & $4-\mathrm{NO}_{2}-\mathrm{C}_{6} \mathrm{H}_{4}$ & $\mathrm{Ph}$ & $\mathrm{Me}$ & 80 & 80 & $129-130$ & $130-131[32]$ \\
\hline $9 \mathrm{k}$ & $\mathrm{Ph}$ & $\mathrm{Ph}$ & Et & 60 & 70 & $166-168$ & $164-166[32]$ \\
\hline 91 & $\mathrm{Ph}$ & $4-\mathrm{Me}-\mathrm{C}_{6} \mathrm{H}_{4}$ & Et & 60 & 80 & $119-121$ & $120-121[32]$ \\
\hline $9 \mathrm{~m}$ & $4-\mathrm{Me}-\mathrm{C}_{6} \mathrm{H}_{4}$ & $\mathrm{Ph}$ & Et & 60 & 85 & $185-186$ & $188-191$ [32] \\
\hline $9 n$ & 4- $\mathrm{Cl}-\mathrm{C}_{6} \mathrm{H}_{4}$ & $\mathrm{Ph}$ & Et & 60 & 80 & $180-182$ & $184-185$ [32] \\
\hline 90 & $4-\mathrm{OMe}-\mathrm{C}_{6} \mathrm{H}_{4}$ & $\mathrm{Ph}$ & Et & 60 & 80 & $174-176$ & 174 [32] \\
\hline
\end{tabular}

A proposed mechanism for the formation of 9 a is shown in scheme 2. Pectin has many free carboxyl group that can active carbonyl group and accelerate the reaction procedure.

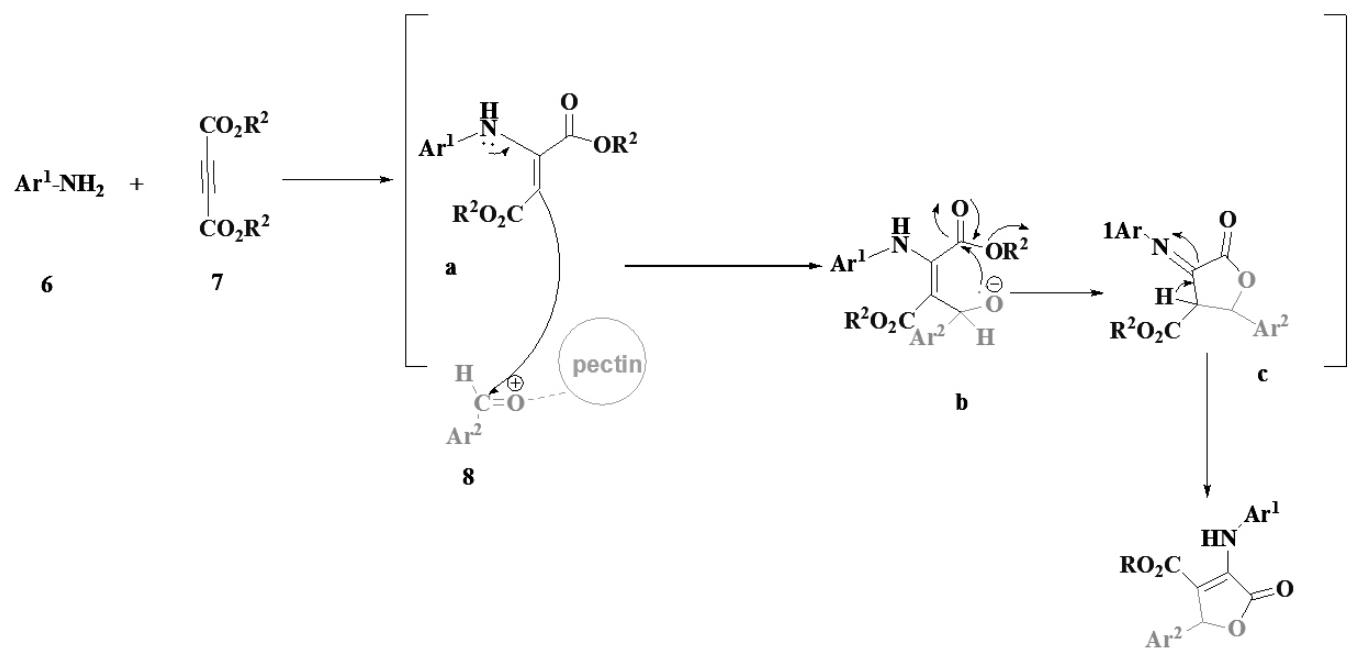

Scheme 2. Proposed mechanism for the synthesis of dihydro-2-oxopyrroles in the presence of pectin as a green catalyst. 
We also investigated the recycling of the catalyst at ambient temperature in aqueous media for the synthesis of 3,4,5-trisubstituted furan-2 $(5 \mathrm{H})$-ones, using a model reaction of 4-methylbenzaldehyde, aniline and dimethyl acetylendicarboxylate. After completion of the reaction, $5 \mathrm{~mL}$ of water was added to the mixture. The pectin was dissolved in water and filtered for separation of the crude product. The separated product was washed with EtOH $(3 \times 2 \mathrm{~mL})$ to give the pure products. In order to recover the catalyst, since pectin is soluble in water, the filtrate was extracted with diethyl ether. The aqueous layer (including pectin) was separated, and its solvent was evaporated under reduced pressure and pectin was recovered and reused. The catalytic system worked well up to five catalytic runs. The recovered catalyst was reused five times without any loss of its activities (Figure 2).

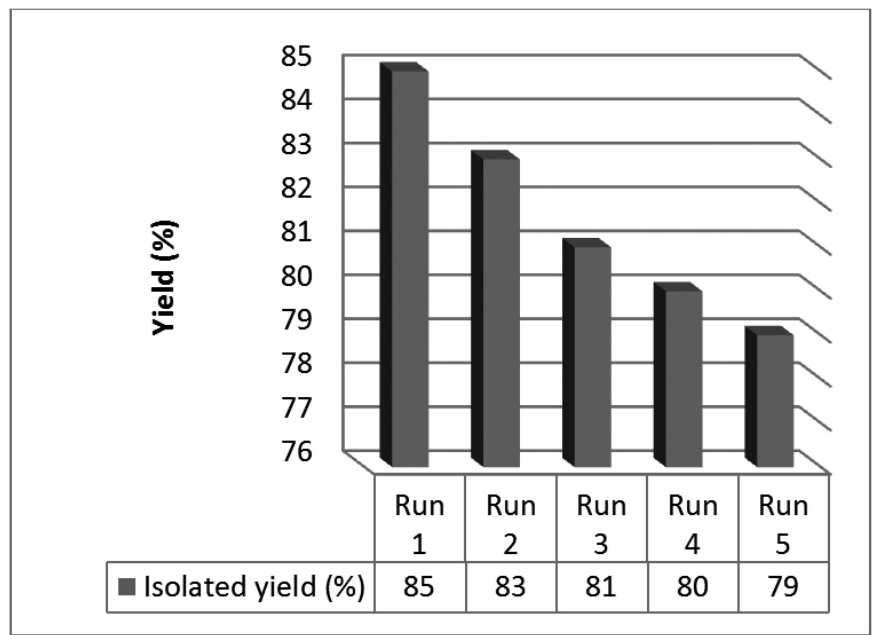

Figure 2. The investigation of the recycling of pectin.

In order to assess the efficiency and generality of this methodology, the obtained result from this methodology has been compared with those of the previously reported methods (Table 4). It was found that the present method is convincingly superior to the reported methods with respect to reaction time and condition and yield of the product. According to the green chemistry law, If possible, synthetic methods should be conducted at ambient temperature and pressure to reduce the energy consume [38]. This methodology has been done at ambient condition for economic and environmental impacts.

Table 4. Comparison of the efficiency of pectin with other reported catalysts in literature.

\begin{tabular}{|c|c|c|c|c|c|}
\hline Entry & Product & $\begin{array}{l}\text { Catalyst/ } \\
\text { Condition }\end{array}$ & Time & $\begin{array}{c}\text { Isolated } \\
\text { yield } \\
(\%)\end{array}$ & References \\
\hline 1 & $5 \mathrm{a}$ & $\begin{array}{c}\mathrm{AcOH} / \mathrm{EtOH}, \\
70^{\circ} \mathrm{C}\end{array}$ & $30 \mathrm{~min}$ & 89 & [39] \\
\hline 2 & $5 a$ & $\mathrm{I}_{2}, \mathrm{MeOH}, \mathrm{r.t}$ & $60 \mathrm{~min}$ & 83 & [40] \\
\hline 3 & $5 a$ & $\begin{array}{c}\mathrm{Al}\left(\mathrm{H}_{2} \mathrm{PO}_{4}\right)_{3}, \\
\mathrm{MeOH}, \mathrm{r} . \mathrm{t}\end{array}$ & $5 \mathrm{~h}$ & 83 & [41] \\
\hline 4 & $5 \mathrm{a}$ & $\begin{array}{c}\text { Pectin, } \\
\mathrm{H}_{2} \mathrm{O}: \text { EtOH, r.t }\end{array}$ & $60 \mathrm{~min}$ & 79 & $\begin{array}{l}\text { Present } \\
\text { work }\end{array}$ \\
\hline 5 & $9 \mathrm{a}$ & $\begin{array}{c}\text { Cyclodextrin / } \\
\mathrm{H}_{2} \mathrm{O}, 60-70{ }^{\circ} \mathrm{C}-\beta\end{array}$ & $12 \mathrm{~h}$ & 78 & [42] \\
\hline 6 & $9 \mathrm{a}$ & $\begin{array}{l}\mathrm{SnCl}_{2}, \mathrm{EtOH}, \\
\text { reflux }\end{array}$ & $7 \mathrm{~h}$ & 86 & [43] \\
\hline 7 & $9 \mathrm{a}$ & $\begin{array}{l}\mathrm{ZnO}, \underset{\mathrm{EtOH}: \mathrm{H}_{2} \mathrm{O}}{90^{\circ} \mathrm{C}} \\
\end{array}$ & $150 \mathrm{~min}$ & 83 & [42] \\
\hline 8 & $9 \mathrm{a}$ & $\begin{array}{c}\text { Pectin, } \\
\mathrm{H}_{2} \mathrm{O}: \text { EtOH, r.t }\end{array}$ & $120 \mathrm{~min}$ & 75 & $\begin{array}{l}\text { Present } \\
\text { work }\end{array}$ \\
\hline
\end{tabular}

\section{EXPERIMENTAL}

\section{Materials and Methods}

IR spectra were obtained on a JASCO FT/IR-460 plusspectrometer. Melting points were taken on an Electrothermal 9100 apparatus. Chemicals were purchased from Merck (Darmastadt,Ge rmany), Acros (Geel, Belgium) and Fluka (Buchs, Switzerland), and used without further purification.

General procedure for the synthesis of dihydro-2-oxopyrroles

A mixture of amine $\mathbf{1}(1 \mathrm{mmol})$ and dialkyl acetylenedicarboxylate $\mathbf{2}$ (1 mmol) in $\mathrm{H}_{2} \mathrm{O}: \mathrm{EtOH}$ (1:3) was stirred for $25 \mathrm{~min}$. Next, amine 3 (1 mmol), formaldehyde 4 ( $37 \%$ solution, $1.5 \mathrm{mmol})$ and pectin $(0.05 \mathrm{~g})$ were added in successively. The reaction mixture was allowed to stir at ambient temperature for appropriate time. After completion of the reaction (monitored by TLC), the precipitate was filtered off and washed with ethanol $(3 \times 2 \mathrm{~mL})$ to give the pure product 5 .

General procedure for the synthesis of 3,4,5-trisubstituted furan2(5H)-ones

A mixture of amine $6(1 \mathrm{mmol})$ and dialkyl acetylenedicarboxylate 7 (1 $\mathrm{mmol})$, aromatic aldehyde $8(1 \mathrm{mmol})$ and pectin $(0.05 \mathrm{~g})$ in $\mathrm{H}_{2} \mathrm{O}: \mathrm{EtOH}(1: 3)$ was stirred at ambient temperature for appropriate time. After completion of the reaction (monitored by TLC), the precipitate was filtered off and washed with ethanol $(3 \times 2 \mathrm{~mL})$ to give the pure product 9 .

Characterization data of some compounds

Methyl 2,5-dihydro-5-oxo-1-phenyl-4-(phenylamino)-1H-pyrrole-3carboxylate (5a). White solid, IR $(\mathrm{KBr})\left(\delta \mathrm{max}, \mathrm{cm}^{-1}\right): 3310(\mathrm{NH}), 1705,1684$, 1645; ${ }^{1} \mathrm{H}$ NMR (400 MHz, $\left.\mathrm{CDCl}_{3}\right): \delta(\mathrm{ppm}): 3.76\left(3 \mathrm{H}, \mathrm{s}, \mathrm{OCH}_{3}\right), 4.57(2 \mathrm{H}, \mathrm{s}$, $\left.\mathrm{CH}_{2}\right), 7.16-7.23(4 \mathrm{H}, \mathrm{m}, \mathrm{ArH}), 7.34(2 \mathrm{H}, \mathrm{t}, \mathrm{J}=8.0 \mathrm{~Hz}, \mathrm{ArH}), 7.42(2 \mathrm{H}, \mathrm{t}, \mathrm{J}=8.0$ $\mathrm{Hz}, \mathrm{ArH}), 7.81(2 \mathrm{H}, \mathrm{d}, \mathrm{J}=8.0 \mathrm{~Hz}, \mathrm{ArH}), 8.05(1 \mathrm{H}, \mathrm{br} \mathrm{s}, \mathrm{NH})$.

Ethyl 4-(p-tolylamino)-2,5-dihydro-5-oxo-1-p-tolyl-1H-pyrrole-3carboxylate (5d). Yellow solid, IR $(\mathrm{KBr})\left(\delta \mathrm{max}, \mathrm{cm}^{-1}\right): 3310(\mathrm{NH}), 1707,1682$, $1649 ;{ }^{\prime} \mathrm{H}$ NMR (400 MHz, $\left.\mathrm{CDCl}_{3}\right): \delta(\mathrm{ppm}): 1.25\left(3 \mathrm{H}, \mathrm{t}, \mathrm{J}=7.2 \mathrm{~Hz}, \mathrm{OCH}_{2} \mathrm{CH}_{3}\right.$ ), $2.36(3 \mathrm{H}, \mathrm{s}, \mathrm{CH}), 2.37(3 \mathrm{H}, \mathrm{s}, \mathrm{CH}), 4.23\left(2 \mathrm{H}, \mathrm{t}, \mathrm{J}=7.2 \mathrm{~Hz}, \mathrm{OCH}_{2} \mathrm{CH}_{3}\right), 4.52$ $\left(2 \mathrm{H}, \mathrm{s}, \mathrm{CH}_{2}\right), 7.06(2 \mathrm{H}, \mathrm{d}, \mathrm{J}=8.4 \mathrm{~Hz}, \mathrm{ArH}), 7.14(2 \mathrm{H}, \mathrm{d}, \mathrm{J}=8.0 \mathrm{~Hz}, \mathrm{ArH}), 7.21$ $(2 \mathrm{H}, \mathrm{d}, \mathrm{J}=8.4 \mathrm{~Hz}, \mathrm{ArH}), 7.69(2 \mathrm{H}, \mathrm{d}, \mathrm{J}=8.8 \mathrm{~Hz}, \mathrm{ArH}), 8.01(1 \mathrm{H}, \mathrm{br} \mathrm{s}, \mathrm{NH}){ }^{13} \mathrm{C}$ NMR (100 MHz, $\left.\mathrm{CDCl}_{1}\right): \delta(\mathrm{ppm}): 14.2,20.9,21.0,48.3,60.2,102.4,119.1$, $122.9,128.9,129.6,134.2,134.6,136.2,136.3,143.1,163.7,164.7$.

Methyl 2,5-dihydro-5-oxo-2-phenyl-4-(phenylamino)furan-3-carboxylate (9a): White solid; IR $(\mathrm{KBr})\left(\delta\right.$ max, $\left.\mathrm{cm}^{-1}\right): 3260,3208,1702,1661 ;{ }^{1} \mathrm{HNMR}(400$ $\left.\mathrm{MHz}, \mathrm{CDCl}_{3}\right): \delta(\mathrm{ppm}): 3.77\left(\mathrm{~s}, 3 \mathrm{H}, \mathrm{OCH}_{3}\right), 5.76(\mathrm{~s}, 1 \mathrm{H}$, benzylic), 7.13 (t, $1 \mathrm{H}, \mathrm{J}=7.3 \mathrm{~Hz}), 7.24-7.31(\mathrm{~m}, 7 \mathrm{H}), 7.52(\mathrm{~d}, 2 \mathrm{H}, \mathrm{J}=8 \mathrm{~Hz}), 8.90$ (br, NH, $1 \mathrm{H}) ;{ }^{13} \mathrm{C}$ NMR(100 MHz, $\left.\mathrm{CDCl}_{3}\right): \delta(\mathrm{ppm}): 165.3$ and 162.7 (ester $\left.\mathrm{CO}\right), 156.3$, 136.1, 134.9,129.0, 128.7, 128.6, 127.4, 125.9, 122.3, 112.8 (aromatic C), 61.6(methoxy C), 52.1 (benzylic C).

Methyl 4-(p-tolylamino)-2,5-dihydro-5-oxo-2-phenylfuran-3-carboxylate (9b): White solid; IR (KBr) $\left(\delta\right.$ max $\left.\mathrm{cm}^{-1}\right): 3228,2950,1706,1677,1513 ;{ }^{1} \mathrm{H}$ NMR (400 MHz,CDCl $)$ : $\delta(\mathrm{ppm}): 2.27$ (s, $\left.3 \mathrm{H}, \mathrm{CH}_{3}\right), 3.76\left(\mathrm{~s}, 3 \mathrm{H}, \mathrm{OCH}_{3}\right), 5.72$ (s, 1H, benzylic), 7.09 (d, 2H, J = 8 Hz), 7.22-7.270 (m, 5H, aromatic), 7.34 $(\mathrm{d}, 2 \mathrm{H}, \mathrm{J}=8.4 \mathrm{~Hz}), 8.86(\mathrm{br}, 1 \mathrm{H}, \mathrm{NH}) ;{ }^{13} \mathrm{C}$ NMR $\left(100 \mathrm{MHz}, \mathrm{CDCl}_{3}\right): \delta(\mathrm{ppm})$ : 165.3and 162.8 (CO of ester), 156.4, 135.8, 135.0, 133.5, 129.6, 128.6,128.5, 127.5, 122.4, 112.6 (C of aromatic), 61.3 (C of methoxy), 52.0 (benzylic $\mathrm{C}$ ), 20.95 (C of methyl).

Ethyl 2-(4-cyanophenyl)-2,5-dihydro-5-oxo-4-(phenylamino)-furan-3carboxylate (9e): White solid; IR (KBr) $\left(\delta\right.$ max, $\left.\mathrm{cm}^{-1}\right): 3293(\mathrm{NH}), 2977,2225$ $(\mathrm{CN}), 1731,1684,1666,1500 ;{ }^{1} \mathrm{H}$ NMR $\left(400 \mathrm{MHz}, \mathrm{CDCl}_{3}\right): \delta(\mathrm{ppm}): 1.23(\mathrm{t}$, $\left.3 \mathrm{H}, \mathrm{J}=7.2 \mathrm{~Hz}, \mathrm{CH}_{3}\right), 4.24$ (q, $\left.2 \mathrm{H}, \mathrm{J}=7.2 \mathrm{~Hz}, \mathrm{CH}_{2}\right), 5.82(\mathrm{~s}, 1 \mathrm{H}$, benzylic), 7.17 $(\mathrm{t}, 1 \mathrm{H}, \mathrm{J}=7.2 \mathrm{~Hz}), 7.32-7.47$ (m, 6H, aromatic), $7.59(\mathrm{~d}, 2 \mathrm{H}, \mathrm{J}=8 \mathrm{~Hz}), 9.03(\mathrm{br}$, $1 \mathrm{H}, \mathrm{NH}) ;{ }^{13} \mathrm{C}$ NMR $\left(100 \mathrm{MHz}, \mathrm{CDCl}_{2}\right): \delta(\mathrm{ppm}): 164.6,162.5$ (CO ofester), $156.89,140.8,135.7,132.5,129.2,128.3,126.3,122.1,118.1,112.6$ (aromatic C), 112.2 (C of CN), 61.6 (methoxy), 60.8 (benzylic), 14.02 ( $\mathrm{CH} 3$ of ethoxy).

\section{CONCLUSION}

In summary, an eco-friendly and straightforward one-pot condensation for the synthesis of dihydro-2-oxopyrroles and 3,4,5-trisubstituted furan$2(5 \mathrm{H})$-ones in the presence of pectin as a highly effective, green, natural and biodegradable catalyst was reported. Pectin is inexpensive, clean, safe, nontoxic, and easy access. Moreover, this method has several other advantages such as, high yields, operational simplicity, reusable catalyst, clean and neutral reaction conditions, which makes it a useful and attractive process for the synthesis of a wide variety of biologically active compounds. 


\section{ACKNOWLEDGMENT}

We gratefully acknowledge financial support from the ResearchCouncil of the University of Sistan and Baluchestan.

\section{REFERENCES}

1. D.Mohnen, In: Barton D, Nakanishi K, Meth-Cohn O (eds) Comprehensive natural products chemistry, Elsevier,Dordrecht, The Netherlands, 497 (1999)

2. M.A. O’Neill, T.Ishii, P. Albersheim, A.G. Darvill, Annu Rev Plant Biol, 55,109 (2004)

3. B.L.Ridley, M.A O’Neill, D.Mohnen, Phytochemistry, 57, 929 (2001)

4. M.A. Laurent, P. Boulenguer, Food Hydrocoll, 17,445 (2003)

5. G.W.Pilgrim, R.H.Walter, D.G. Oakenfull, In: Walters RH(ed) The chemistry and technology of pectin. Academic Press.Inc., San Diego, 23 (1991)

6. W. Pilnik, A.G.J. Voragen, In: Hulme HC (ed) The biochemistry of fruits and their products, Academic Press, London, 1, 53 (1970)

7. A. Akansha, A.Deepali, B.Anamika, K.Virendra Kumar, Res. J. Recent. Sci., 364 (2014)

8. W.Shi, Y.Duan, Y.Qian, M.Li, L.Yang, W. Hu, Med. Chem. Lett. 20, 3592 (2010)

9. X.Luo, M.Shu, Y.Wang, J.Liu, W.Yang, Z.Lin, 3D-QSAR .Molecules. 17, 2015 (2012)

10. J. W.Lampe, Y. L.Chou, R. G.Hanna, S. V. D.Meo, P. W. Erhardt, A A.Hagedorn, Ingebretsen, W. R.; Cantor, E. J. Med. Chem. 36, 1041 ( 1993)

11. T.Kawasuji, M.Fuji, T.Yoshinaga, A.Sato, T. Fujiwara, R.Kiyama, Bioorg. Med. Chem. 15, 5487 (2007)

12. C.Peifer, R.Selig, K.Kinkel, D.Ott, F.Totzke, C.Schächtele, R.Heidenreich, M. Röcken, D.Schollmeyer, S. Laufer, J. Med.Chem. 51, 3814 (2008)

13. T.Bach, H.Brummerhop, Angew. Chem., Int. Ed. 37, 3400 (1998)

14. M.Kawase, M. Hirabayashi, S.Saito, K.Yamamoto, Tetrahedron Lett. 40 , 2541 (1999)

15. D.M.P, Y.M, H.W, G.LS., J. Am. Chem. Soc. 125, 4692 (2003)

16. A. J.Clark, C. P. M.C.Dell, , J. M. Donagh, J.Geden, P.Mawdsley, Org. Lett. 5, 2063 (2003)

17. J.Chen, P.Q.Huang, Y.Queneau, J. Org. Chem. 74,7457 (2009)

18. H.He, H. Y.Yang, R.Bigelis, E. H.Solum, M.Greenstein, G. T. Carter, Tetrahedron Lett. 43, 1633 (2002)

19. T.Agatsuma, T. Akama, S.Nara, S.Matsumiya, R.Nakai, H.Ogawa, S.Otaki, S.I.Ikeda, Y.Saitoh, Y. Kanda, Org. Lett. 4, 4387 (2002)

20. M. Adib, M. Mahdavi, M. A.Noghani, H. R. Bijanzadeh, Tetrahedron Lett. 48, 8056 (2007)

21. M.Aginagalde, T.Bello, C.Masdeu, Y.Vara, A.Arrieta, F. P. Cossío, J. Org. Chem. 75, 7435 (2010)

22. F.Palacios, J.Vicario, D. Aparicio, Eur. J. Org. Chem. 2843 (2006)

23. J.Zhang, P. G.Blazecka, J. G. Davidson, Org. Lett. 5,553 (2003)

24. M.J.Fan, B.Qian, L.B.Zhao, Y.M. Liang, Tetrahedron. 63, 8987 (2007)

25. Q.Zhu, H.Jiang, J.Li, S.Liu, C.Xia, M. Zhang, J. Comb. Chem. 11, 685 (2009)

26. A. T.Khan, A.Ghosh, M.d. M. Khan, Tetrahedron Lett. 53, 2622 (2012)

27. G. J.Hollingworth, G.Perkins, J. B.Sweeney, J. Chem. Soc., Perkin Trans. $1,913(1996)$

28. R.Mabon, A. M. E. Richecoeur, J. B. Sweeney, J. Org. Chem. 64, 328 (1999)

29. R. Mabon, A. M. E.Richecoeur, J. B. Sweeney, Tetrahedron. 58, 9117 (2002)

30. M.Bassetti, A. D'Annibale, A.Fanfoni, F. Minissi, Org. Lett. 7, 1805 (2005)

31. A.Yazdani Elah Abadi, M.T. Maghsoodlou, R.Heydari, R. Mohebat, Res. Chem.Int. doi: 10.1007/s11164-015-2083-5(2015)

32. M.Kngani, N.Hazeri, M.T. Maghsoodlou, J.Saud.Chem.Soc. doi:10.1016/j. jscs.2015.03.002 (2015)

33. A.Moradi, R.Heydari, M.T. Maghsoodlou, Res.Chem.Int. doi: 10.1007/ s11164-014-1818-z (2014)

34. N.Hazeri, S. S.Sajadikhah, M. T.Maghsoodlou, S.Mohamadian-Souri, M.Norouzi, M. Moein, Chin.Chem.Soc. 61,217 (2013)

35. S. S.Sajadikhah, N.Hazeri, M. T. Maghsoodlou, S. M. Habibi-Khorassani, Chin.Chem.Soc. 60, 1003 (2013)

36. N.Hazeri, M.T. Maghsoodlou, S. M.Habibi-Khorassani, J. Aboonajmi, S. S. Sajadikhah, Chin.Chem.Soc. 60, 355 (2013)
37. R.Doostmohammadi, M.T.Maghsoodlou, N.Hazeri, S.M. HabibiKhorassani, Chin.Chem.lett, 24, 901 (2013)

38. P. T. Anastas, J. C. Warner, Oxford university press (2000)

39. Q.Zhu, H.Jiang, J. Li, ,S.Liu, C.Xia, , M. Zhang, J.Com. Chem., 11, 685 (2009)

40. A. T.Khan, A.Ghosh, M. M.Khan, Tetrahedron Lett. 53, 2622 (2012)

41. S. S.Sajadikhah, N.Hazeri, M. T.Maghsoodlou, S. M. Habibi-Khorassani, A.Beigbabaei, A. C.Willis, J.Iran. Chem. Soc. 10, 863 (2013).

42. S. N.Murthy, B.Madhav, A. V.Kumar, K. R. Rao, Y. V. D. Nageswar, Tetrahedron, 65 , 5251 (2009)

43. L.Nagarapu, U. N.Kumar, P. Upendra, R. Bantu, Syn. Commu. 42, 2139 (2012) 